\title{
Binary Field Multiplication on ARMv8
}

\author{
Hwajeong Seo ${ }^{1}$, Zhe Liu ${ }^{2}$, Yasuyuki Nogami ${ }^{3}$, \\ Jongseok $\mathrm{Choi}^{1}$, and Howon Kim ${ }^{1 \star}$ \\ 1 Pusan National University, \\ School of Computer Science and Engineering, \\ San-30, Jangjeon-Dong, Geumjeong-Gu, Busan 609-735, Republic of Korea \\ \{hwajeong, jschoi85, howonkim\}@pusan.ac.kr \\ 2 University of Luxembourg, \\ Laboratory of Algorithmics, Cryptology and Security (LACS), \\ 6, rue R. Coudenhove-Kalergi, L-1359 Luxembourg-Kirchberg, Luxembourg \\ \{zhe.liu\}@uni.lu \\ 3 Okayama University, \\ Graduate School of Natural Science and Technology, \\ 3-1-1, Tsushima-naka, Kita, Okayama, 700-8530, Japan \\ \{yasuyuki.nogami\}@okayama-u.ac.jp
}

\begin{abstract}
In this paper, we show efficient implementations of binary field multiplication over ARMv8. We exploit an advanced 64-bit polynomial multiplication (PMULL) supported by ARMv8 and conduct multiple levels of asymptotically faster Karatsuba multiplication. Finally, our method conducts binary field multiplication within 57 clock cycles for B-251. Our proposed method on ARMv8 improves the performance by a factor of 5.5 times than previous techniques on ARMv7.
\end{abstract}

Keywords: Polynomial Multiplication, Binary Field Multiplication, ARMv8, Elliptic Curve Cryptography, Karatsuba Multiplication

\section{Introduction}

Since binary field multiplication is an important component of elliptic curve cryptography and authenticated encryption, many researches have studied the high speed implementation of binary field multiplication in software engineering. The typical binary field multiplication over embedded processor may compute the results with bitwise-xor and logical shift operations. The other more clever approach exploits the look-up table by calculating the part of results in advance $[6,7,10,8,9]$. Recently, many modern embedded processors adopt the advanced built-in binary field multiplication. ARMv7 supports VMULL.P8 operation which can compute eight 8-bit wise polynomial multiplications with single instruction. In [2], author shows that efficient implementation techniques to construct the 64-bit binary field multiplication with the VMULL.P8 operation. After then multiple levels of Karatsuba multiplication is applied to several binary field multiplications including $\mathbb{F}_{2^{251}}, \mathbb{F}_{2^{283}}$ and $\mathbb{F}_{2^{571}}$. The most recent processor, ARMv8,

\footnotetext{
* Corresponding Author
} 
supports PMULL operation which can compute 64-bit wise polynomial multiplication with single instruction. In [3], author shows that compact implementation of GCM based authenticated encryption with the PMULL operation. Since the 64-bit multiplication is quite fast enough for 128-bit multiplication, they avoid Karatsuba multiplication. The detailed 128-bit polynomial multiplication is available in Algorithm 1. The implementations achieved 11 times faster results than ARMv7. However, the paper does not show binary field multiplication for long length operands. The long operands are required to compute ECC based cryptography. In this paper, we present efficient implementations of long bits binary field multiplication. We applied multiple levels of Karatsuba multiplication and achieved 5.5 times faster than ARMv7 implementations.

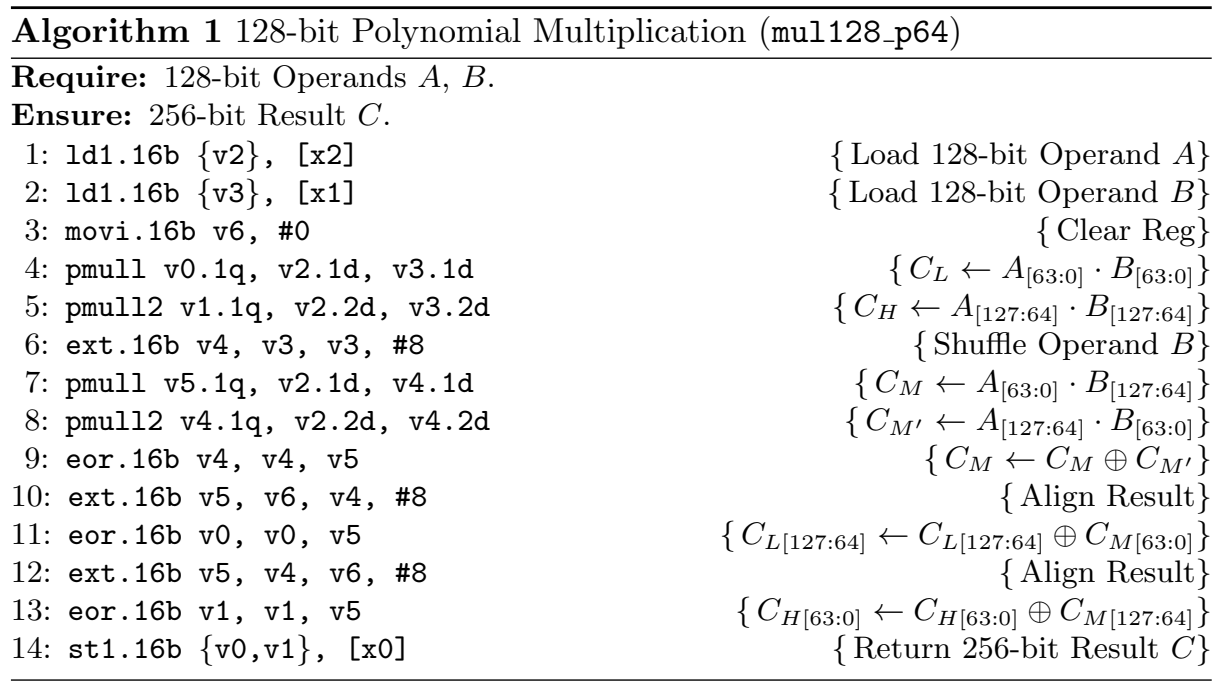

The remainder of this paper is organized as follows. In Section 2, we recap the target ARM processor and Karatsuba method. In Section 3, we propose the efficient binary field multiplication. In Section 4, we evaluate the performance of proposed methods in terms of clock cycles. Finally, Section 5 concludes the paper.

\section{Related Works}

\subsection{ARM Processor}

ARM processor is a well known family of RISC processor architectures introduced in 1985 [11]. The most recent version, ARMv8, supports both 32-bit and 64-bit processing. The 32-bit ARMv8 architecture is known as AArch32, while 
the 64-bit is known as AArch64. An ARMv8 processor can support both, allowing the execution of 32-bit and 64-bit applications. ARM processors support a single-instruction multiple-data (SIMD) module called the NEON engine. AArch32 features sixteen 32-bit registers (R0-R15) and sixteen 128-bit NEON registers (Q0-Q15). The NEON registers can also be viewed as pairs of 64-bit registers (D0-D32). For example, D0 and D1 are the lower and higher parts of Q0, respectively. AArch64 features thirty two 64-bit registers (X0-X31) and thirty two 128-bit NEON registers (V0-V31). The NEON registers can no longer be viewed as pairs of 64-bit registers. From ARMv8, two polynomial dedicated instructions, PMULL and PMULL2, are available. Both of which carry out a single 64-bit multiplication. In both cases, the inputs are 128-bit registers. Their difference is that in PMULL the lower 64-bit parts of the inputs are used as operands, while in PMULL2 the higher 64-bit parts are used [3].

\subsection{Karatsuba Algorithm}

The basic idea of Karatsuba multiplication is to split a multiplication of two $s$ words operands into three multiplications of size $\frac{s}{2}$, which is possible at the expense of some additions [5]. Taking the multiplication of $s$ words operands $A$ and $B$ as an example, we represent the operands as $A=A_{H} \cdot 2^{\frac{s}{2}}+A_{L}$ and $B=B_{H} \cdot 2^{\frac{s}{2}}+B_{L}$. The multiplication $P=A \cdot B$ can be computed according to the Equation 1.

$$
A_{H} \cdot B_{H} \cdot 2^{s}+\left[\left(A_{H}+A_{L}\right)\left(B_{H}+B_{L}\right)-A_{H} \cdot B_{H}-A_{L} \cdot B_{L}\right] \cdot 2^{\frac{s}{2}}+A_{L} \cdot B_{L}
$$

Karatsuba method roughly executes $\frac{3 s^{2}}{4}$ mul instructions to multiply two $s$ word operands [4]. Recently, the refined Karatsuba's algorithm from a Crypto 2009 paper by Bernstein [1] makes efficient use of the available registers to keep the low overheads from load and store instructions.

\section{Proposed Method}

\subsection{Polynomial Multiplication}

Polynomial multiplication can be implemented in ordinary or Karatsuba method. The Karatsuba multiplication is an efficient approach when size of operand is long enough than processor's word. Since the ARMv8 processor is 64-bit architecture, several parameters are not favorable with Karatsuba multiplication. In this section, we investigate the proper size of minimum Karatsuba technique.

Four and Eight Terms of Multiplications The efficient Karatsuba multiplication techniques are highly relied on the number of terms where term is (operand size/word size). In this section we firstly explore the two, four and eight terms of multiplications. For two terms as studied in [3], ordinary multiplication 
is efficient than Karatsuba approach. As we can see the comparison results in Table 1. Karatsuba method can reduce the number of multiplication by 1 . However, more number of other instructions including eor and ext are required. For this reason, ordinary multiplication is better choice for 128-bit polynomial multiplication.

Table 1. Comparison of 128-bit polynomial multiplication methods

\begin{tabular}{ccccc}
\hline Instructions & pmull & eor & movi & ext \\
\hline Ordinary & 4 & 3 & 1 & 3 \\
Karatsuba & 3 & 6 & 1 & 4 \\
\hline
\end{tabular}

In case of long operands (256, 512 and etc), Karatsuba approach is better choice, because total number of instructions are smaller than ordinary approach. We use the Karatsuba algorithm for both cases and our solution is a combination of the Karatsuba algorithm and a multiplier based on PMULL which we have named the Karatsuba/NEON/PMULL multiplier (KNP).

Four terms (256-bit) polynomial multiplication is executed with 1 level of Karatsuba multiplication. The inner 128-bit multiplication is readily established with Algorithm 1. The detailed four terms polynomial multiplication is available in Algorithm 2. In Step 1 and 2, 256-bit operands are loaded from memory. In Step 3 and 4, lower parts of operands are bit-wise exclusive-ored with higher parts. In Step 5, 128-bit register (v14) is cleared. In Step 6, 7 and 8, three 128bit wise polynomial multiplications $\left(C_{L} \leftarrow A_{L} \cdot B_{L}\right.$ and $C_{H} \leftarrow A_{H} \cdot B_{H}$ and $\left.C_{M} \leftarrow\left(A_{L} \oplus A_{H}\right) \cdot\left(B_{L} \oplus B_{H}\right)\right)$ are conducted. The first two input variables of mul128_p64 are results and third and fourth variables represent operands and the last three operands indicate temporal storages. From Step 9 to 14, intermediate results are bit-wise exclusive-ored. At step 15, total 512-bit results are stored into memory.

Eight terms (512-bit) polynomial multiplication consists of 2 levels of Karatsuba multiplication. The first level consists of 256-bit polynomial multiplications and the multiplication is following the Algorithm 2. The detailed 512-bit multiplication is written in Algorithm 3. In Step 1 and 2, 256-bit operands are loaded from memory. In Step 3 and 6, lower parts of operands are bit-wise exclusiveored with higher parts. In Step 7, 128-bit register (v14) is cleared. In Step 8, 9 and 10, three 128-bit wise polynomial multiplications $\left(C_{L} \leftarrow A_{L} \cdot B_{L}\right.$ and $C_{H} \leftarrow A_{H} \cdot B_{H}$ and $\left.C_{M} \leftarrow\left(A_{L} \oplus A_{H}\right) \cdot\left(B_{L} \oplus B_{H}\right)\right)$ are conducted. The first two input variables of mul256_p64 are results and third and fourth variables represent operands and the last three operands indicate temporal storages. From Step 11 to 22, intermediate results are bit-wise exclusive-ored. At step 23 and 24 , total 1024-bit results are stored into memory.

Three and Six of Multiplications In this section we explore the three, six and nine terms of multiplications. For three terms, ordinary multiplication is 


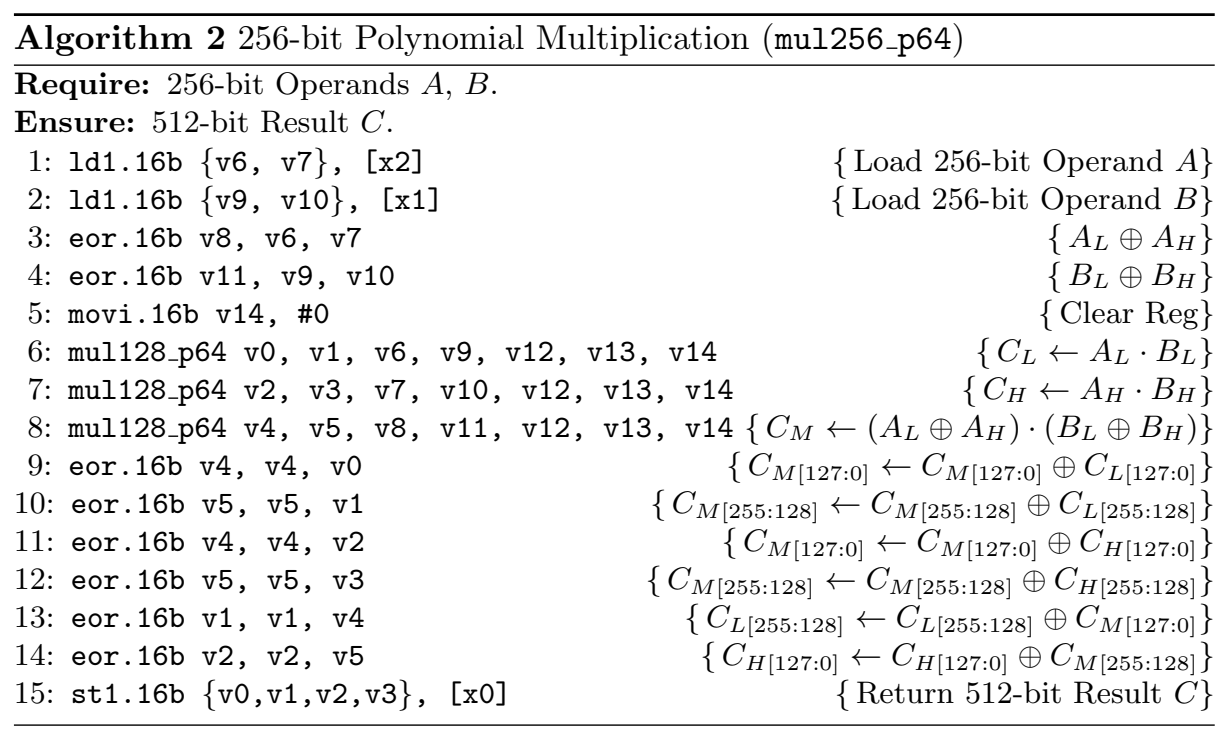

the more efficient method than that of Karatsuba. The comparison results are drawn in Table 2. Three terms of Karatsuba multiplication reduces the number of multiplication from 9 to 6 . However, additional 8 and 5 times of eor and ext instructions are required. For this reason, ordinary multiplication is better choice for 192-bit polynomial multiplication.

Table 2. Comparison of 192-bit polynomial multiplication methods

\begin{tabular}{ccccc}
\hline Instructions & pmull & eor & movi & ext \\
\hline Ordinary & 6 & 16 & 1 & 8 \\
Karatsuba & 9 & 7 & 1 & 3 \\
\hline
\end{tabular}

The detailed three terms of polynomial multiplication is available in Algorithm 4. In Step 1 and 2, 192-bit operands ( $A$ and $B$ ) are loaded from memory. By using option $8 \mathrm{~b}$, we loaded operands by sequential 64-bit format to the 128-bit registers, which lefts higher 64-bit as an empty. In Step $3 \sim 6$, four multiplications including $\left(C_{L} \leftarrow A_{[63: 0]} \cdot B_{[63: 0]}, T_{L} \leftarrow A_{[63: 0]} \cdot B_{[127: 64]}\right.$, $C_{M} \leftarrow A_{[63: 0]} \cdot B_{[191: 128]}$ and Temp $\left.\leftarrow A_{[127: 64]} \cdot B_{[63: 0]}\right)$ are conducted. After then results $\left(A_{[127: 64]} \cdot B_{[63: 0]}\right)$ are added to $T_{L}$. From Step 8 to 15 , remaining multiplications are conducted and then added to the intermediate results. After then the results are aligned and accumulated to the intermediate results in Step $17 \sim 22$. Finally, total 384-bit results are stored into memory.

For six terms of polynomial multiplication, we firstly split six terms largely into two parts consisting of three terms. The two part is computed with 1 level of Karatsuba multiplication. The inner three-term of multiplication is executed 


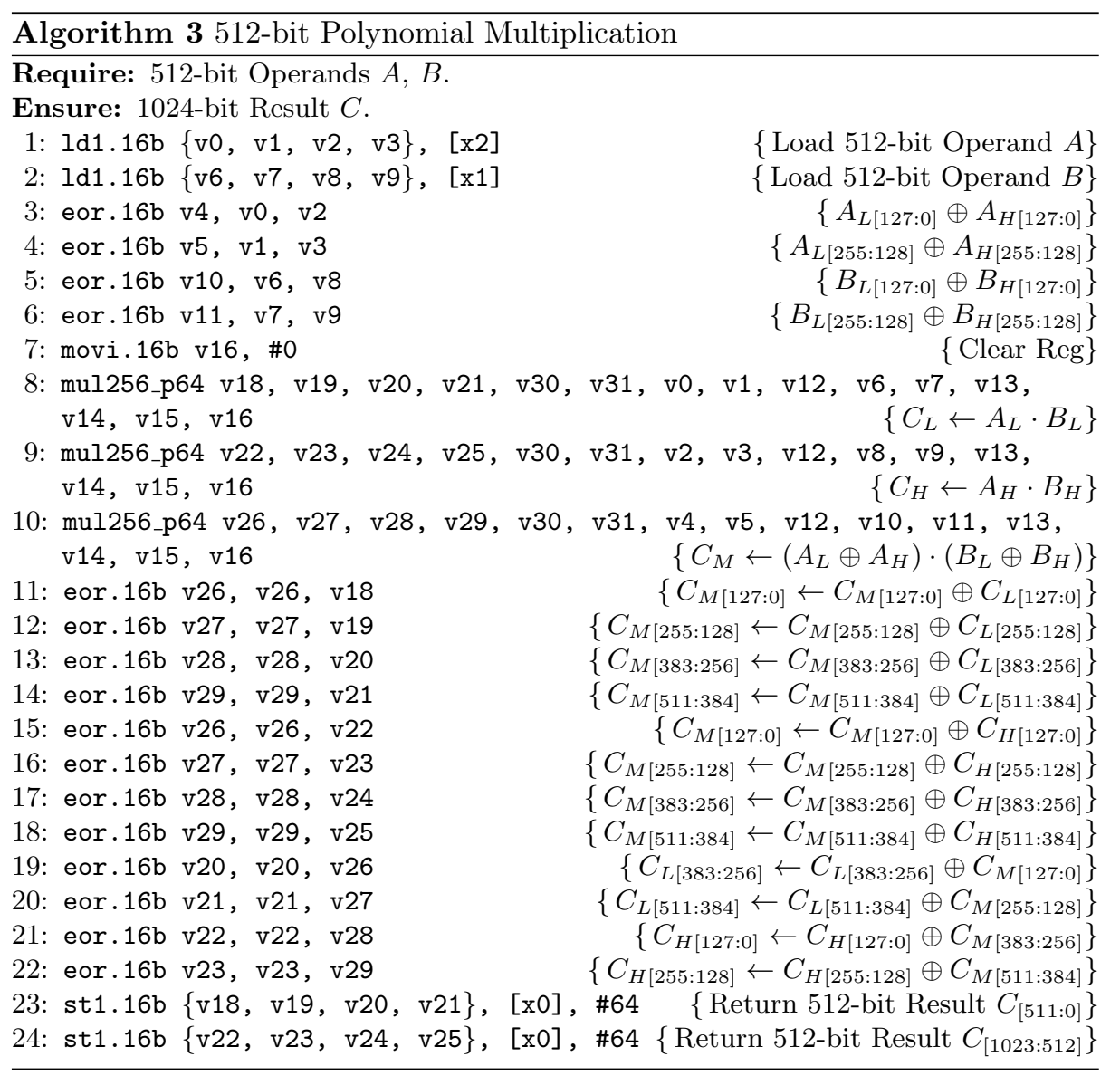




\begin{tabular}{|c|c|}
\hline Algorithm 4 192-bit Polynom & tion (mul192_p64) \\
\hline Require: 192-bit Operands $A, B$. & \\
\hline Ensure: 384 -bit Result $C$. & \\
\hline 1: $\mathrm{ld} 1.8 \mathrm{~b}\{\mathrm{v} 0, \mathrm{v} 1, \mathrm{v} 2\},[\mathrm{x} 2]$ & $\{$ Load 192-bit Operand $A\}$ \\
\hline 2: $1 \mathrm{~d} 1.8 \mathrm{~b}\{\mathrm{v} 3, \mathrm{v} 4, \mathrm{v} 5\},[\mathrm{x} 1]$ & $\{$ Load 192-bit Operand $B\}$ \\
\hline 3: pmull v6.1q, v0.1d, v3.1d & $\left\{C_{L} \leftarrow A_{[63: 0]} \cdot B_{[63: 0]}\right\}$ \\
\hline 4: pmull v9.1q, v0.1d, v4.1d & $\left\{T_{L} \leftarrow A_{[63: 0]} \cdot B_{[127: 64]}\right\}$ \\
\hline 5: pmull v7.1q, v0.1d, v5.1d & $\left\{C_{M} \leftarrow A_{[63: 0]} \cdot B_{[191: 128]}\right\}$ \\
\hline 6: pmull v11.1q, v1.1d, v3.1d & $\left\{T e m p \leftarrow A_{[127: 64]} \cdot B_{[63: 0]}\right\}$ \\
\hline 7: eor.16b v9, v9, v11 & $\left\{T_{L} \leftarrow T_{L} \oplus T e m p\right\}$ \\
\hline 8: pmull v11.1q, v1.1d, v4.1d & $\left\{T e m p \leftarrow A_{[127: 64]} \cdot B_{[127: 64]}\right\}$ \\
\hline 9: eor. $16 \mathrm{~b}$ v7, v7, v11 & $\left\{C_{M} \leftarrow C_{M} \oplus T e m p\right\}$ \\
\hline 10: pmull v10.1q, v1.1d, v5.1d & $\left\{T_{H} \leftarrow A_{[127: 64]} \cdot B_{[191: 128]}\right\}$ \\
\hline 11: pmull v11.1q, v2.1d, v3.1d & $\left\{T e m p \leftarrow A_{[191: 128]} \cdot B_{[63: 0]}\right\}$ \\
\hline 12: eor. $16 \mathrm{~b}$ v7, v7, v11 & $\left\{C_{M} \leftarrow C_{M} \oplus T e m p\right\}$ \\
\hline 13: pmull v11.1q, v2.1d, v4.1d & $\left\{T e m p \leftarrow A_{[191: 128]} \cdot B_{[127: 64]}\right\}$ \\
\hline 14: eor. $16 \mathrm{~b}$ v10, v10, v11 & $\left\{T_{H} \leftarrow T_{H} \oplus T e m p\right\}$ \\
\hline 15: pmull v8.1q, v2.1d, v5.1d & $\left\{C_{H} \leftarrow A_{[191: 128]} \cdot B_{[191: 128]}\right\}$ \\
\hline 16: movi.16b v11, \#0 & $\{$ Clear Reg $\}$ \\
\hline 17: ext.16b v11, v11, v9, \#8 & $\{$ Align Result $\}$ \\
\hline 18: ext.16b v9, v9, v10, \#8 & $\{$ Align Result $\}$ \\
\hline 19: ext.16b v10, v10, v11, \#8 & $\{$ Align Result $\}$ \\
\hline 20: eor. $16 \mathrm{~b}$ v6, v6, v11 & $\left\{C_{L[127: 64]} \leftarrow C_{L[127: 64]} \oplus T_{L[63: 0]}\right\}$ \\
\hline 21: eor.16b v7, v7, v9 & $\left\{C_{M} \leftarrow C_{M} \oplus\left\{T_{H[63: 0]} \| T_{L[127: 64]}\right\}\right\}$ \\
\hline 22: eor. $16 \mathrm{~b}$ v8, v8, v10 & $\left\{C_{H[63: 0]} \leftarrow C_{H[63: 0]} \oplus T_{H[127: 64]}\right\}$ \\
\hline 23: st1.16b $\{\mathrm{v} 6, \mathrm{v} 7, \mathrm{v} 8\},[\mathrm{x} 0]$ & $\{$ Return 384-bit Result $C\}$ \\
\hline
\end{tabular}


with ordinary multiplication described in Algorithm 4. The detailed six terms of polynomial multiplication is available in Algorithm 5. In Step $1 \sim 4$, 384-bit operands are loaded from memory. In Step $5 \sim 10$, lower parts of operands are bit-wise exclusive-ored with higher parts. In Step 11, 128-bit register (v14) is cleared. In Step 12, 13 and 14, three 192-bit wise polynomial multiplications $\left(C_{L} \leftarrow A_{L} \cdot B_{L}\right.$ and $C_{H} \leftarrow A_{H} \cdot B_{H}$ and $\left.C_{M} \leftarrow\left(A_{L} \oplus A_{H}\right) \cdot\left(B_{L} \oplus B_{H}\right)\right)$ are conducted. The first six input variables of mul192_p64 are operands and seventh, eighth and nine-th variables represent temporal storages and the last three operands indicate results. From Step 15 to 28, intermediate results are bit-wise exclusive-ored. At step 29 and 30, total 768-bit results are stored into memory.

\subsection{Binary Field Multiplication}

$s$ word of binary field multiplication produce values of degree at most $2 s-2$, which must be reduced modulo $f(z)=z^{m}+r(z)$. The usual approach is to multiply the higher parts by $r(z)$ using shift and xors. For small polynomials $r(z)$ we can exploit the PMULL instruction to carry out 64 -bit multiplication by $r(z)$.

Curve B-251 The modulo of binary field $\mathbb{F}_{2^{251}}$ is defined by $\left(r(z)=z^{7}+z^{4}+\right.$ $\left.z^{2}+1\right)$. The detailed reduction method is available in Algorithm 6. In Step 1 $\sim 3$, intermediate results are shifted by 59 to the right. In Step 4 and 5, $60 \sim$ 64 -th bits are cleared. In Step 6 and 7, intermediate results are shifted by 5 to the left. In Step $8 \sim 10$, the results are aligned and then the shifted results are accumulated. In Step 13 and 14, the lower 8-bit of register v15 is set to 0x95. In Step $15 \sim 19$, the higher intermediate results are multiplied by $r(z)$. In Step 20 $\sim 27$, the computed results are aligned and then accumulated to intermediate results. From Step 28 to 41, one more round of reduction process is conducted.

\section{Evaluation}

In order to test ARMv8 instruction set, we set the development environment as follows. We used Xcode (ver 6.3.2) as a development IDE and tested the program over iPad Mini2 (iOS 8.4). The iPad Mini2 supports Apple A7 with 64-bit architecture operated in 1.3GHz. In Table 3, we show performance results onv various length of polynomial multiplications. For 192-bit, we used ordinary multiplication and the other parameters exploit the Karatsuba multiplication.

In Table 4, the comparison results of binary field multiplication is available. Previous works by [2] uses KNV method. This method is quiet slow because it adopts eight vectorized 8-bit polynomial multiplication namely VMULL. Unlike previous works, we exploit new 64-bit polynomial multiplication namely PMULL. This method significantly improves the performance by a factor of 5.5 times than previous works. However, we couldn't compare our results with the methods on same ARMv8 architecture because this is the first implementation for ECC 


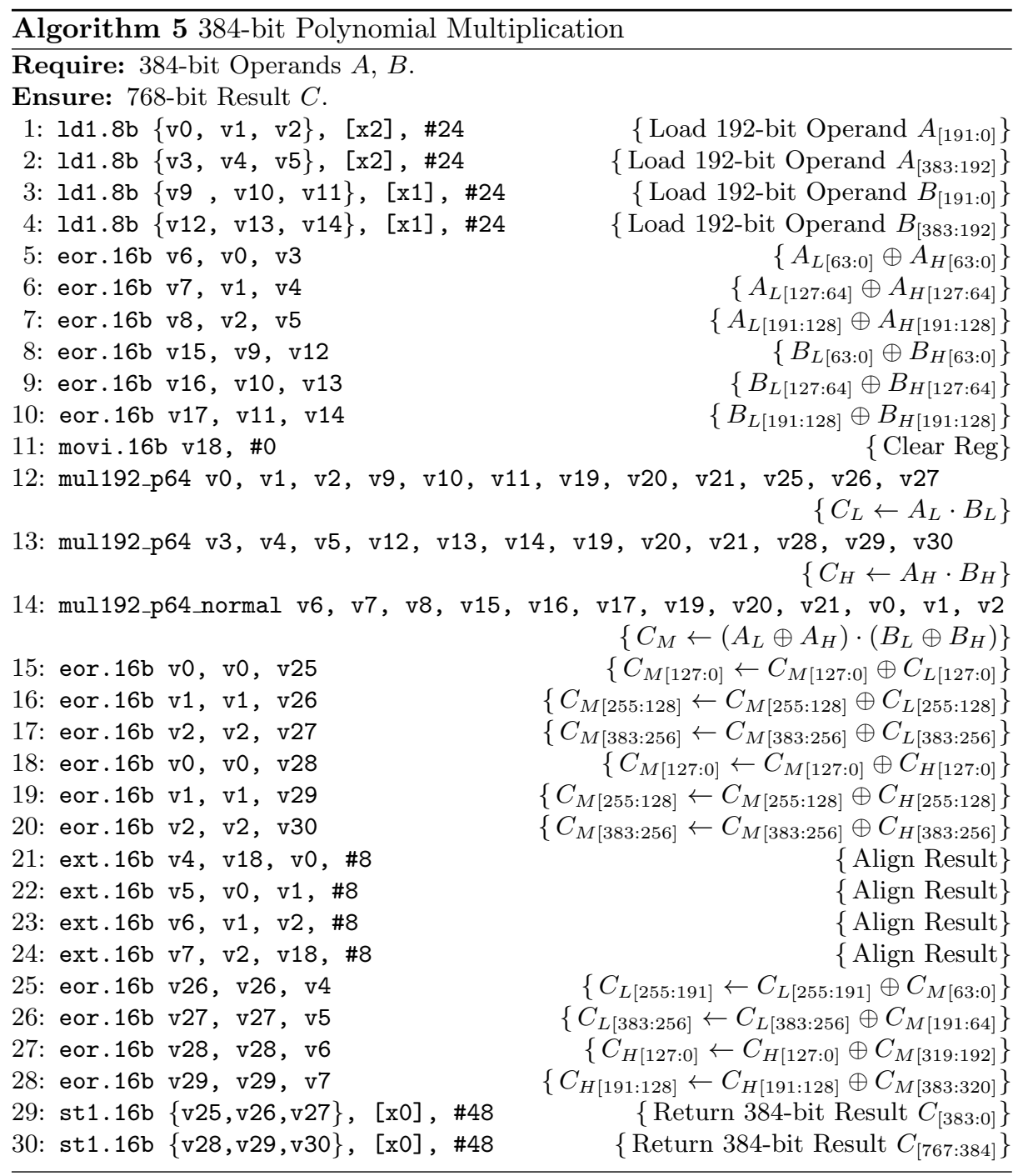

Table 3. Performance of polynomial multiplication, (2): two-term

\begin{tabular}{ccccc}
\hline Length & 192 & 256 & 384 & 512 \\
\hline Karatsuba & - & 1-level(2) & 1-level(2) & 2-level(2) \\
Clock Cycles & 11 & 21 & 49 & 75 \\
\hline
\end{tabular}




\begin{tabular}{|c|c|}
\hline Algorithm 6 251-bit Polynomial & \\
\hline Require: 502-bit Operands $A$. & \\
\hline Ensure: 251-bit Result $C$. & \\
\hline 1: ushr.2d v4, v1, \#59 & $\{$ Right Shift by \#59\} \\
\hline 2: ushr.2d v5, v2, \#59 & $\{$ Right Shift by \#59\} \\
\hline 3: ushr.2d v6, v3, \#59 & $\{$ Right Shift by \#59\} \\
\hline 4: $\operatorname{shl} .2 \mathrm{~d}$ v1, v1, \#5 & $\{$ Clear High Bits $\}$ \\
\hline 5: ushr.2d v1, v1, \#5 & $\{$ Clear High Bits $\}$ \\
\hline 6: $\operatorname{shl} .2 \mathrm{~d}$ v7, v2, \#5 & $\{$ Left Shift by $\# 5\}$ \\
\hline 7: shl.2d v8, v3, \#5 & $\{$ Left Shift by $\# 5\}$ \\
\hline 8: ext.16b v4, v4, v5, \#8 & $\{$ Align Result $\}$ \\
\hline 9: ext.16b v5, v5, v6, \#8 & $\{$ Align Result\} \\
\hline 10: ext.16b v6, v6, v14, \#8 & $\{$ Align Result\} \\
\hline 11: eor. $16 \mathrm{~b} v 4, \mathrm{v} 4, \mathrm{v} 7$ & $\{$ Accumulate Intermediate Result $\}$ \\
\hline 12: eor. $16 \mathrm{~b}$ v5, v5, v8 & $\{$ Accumulate Intermediate Result $\}$ \\
\hline 13: movi.16b v15, \#149 & $\{$ Set $0 \times 95\}$ \\
\hline 14: ushr.2d v15, v15, \#56 & $\{$ Clear High 56-bit $\}$ \\
\hline 15: pmull v16.1q, v4.1d, v15.1d & $\{$ Multiply Modulo $\}$ \\
\hline 16: pmull2 v17.1q, v4.2d, v15.2d & $\{$ Multiply Modulo $\}$ \\
\hline 17: pmull v18.1q, v5.1d, v15.1d & $\{$ Multiply Modulo\} \\
\hline 18: pmull2 v19.1q, v5.2d, v15.2d & $\{$ Multiply Modulo\} \\
\hline 19: pmull v20.1q, v6.1d, v15.1d & $\{$ Multiply Modulo\} \\
\hline 20: ext.16b v21, v14, v17, \#8 & $\{$ Align Result $\}$ \\
\hline 21: ext.16b v22, v17, v19, \#8 & $\{$ Align Result \\
\hline 22: ext.16b v23, v19, v14, \#8 & $\{$ Align Result $\}$ \\
\hline 23: eor. $16 \mathrm{~b}$ v16, v16, v21 & $\{$ Accumulate Intermediate Result $\}$ \\
\hline 24: eor. $16 \mathrm{~b}$ v18, v18, v22 & $\{$ Accumulate Intermediate Result $\}$ \\
\hline 25: eor. $16 \mathrm{~b}$ v2, v20, v23 & $\{$ Accumulate Intermediate Result $\}$ \\
\hline 26: eor. $16 \mathrm{~b}$ v0, v0, v16 & $\{$ Accumulate Intermediate Result $\}$ \\
\hline 27: eor. $16 \mathrm{~b}$ v1, v1, v18 & $\{$ Accumulate Intermediate Result $\}$ \\
\hline 28: ushr.2d v4, v1, \#59 & $\{$ Right Shift by \#59\} \\
\hline 29: ushr.2d v5, v2, \#59 & $\{$ Right Shift by \#59\} \\
\hline 30: shl.2d v1, v1, \#5 & $\{$ Clear High Bits $\}$ \\
\hline 31: ushr.2d v1, v1, \#5 & $\{$ Clear High Bits $\}$ \\
\hline 32: $\operatorname{shl} .2 \mathrm{~d}$ v7, v2, \#5 & $\{$ Left Shift by $\# 5\}$ \\
\hline 33: ext.16b v4, v4, v5, \#8 & $\{$ Align Result $\}$ \\
\hline 34: eor. $16 b$ v4, v4, v7 & $\{$ Accumulate Intermediate Result $\}$ \\
\hline 35: pmull v16.1q, v4.1d, v15.1d & $\{$ Multiply Modulo\} \\
\hline 36: $\mathrm{pmull2}$ v17.1q, v4.2d, v15.2d & $\{$ Multiply Modulo\} \\
\hline 37: ext. $16 \mathrm{~b}$ v21, v14, v17, \#8 & $\{$ Align Result $\}$ \\
\hline 38: ext.16b v22, v17, v14, \#8 & $\{$ Align Result $\}$ \\
\hline 39: eor. $16 \mathrm{~b}$ v0, v0, v16 & $\{$ Accumulate Intermediate Result $\}$ \\
\hline 40: eor. $16 \mathrm{~b}$ v0, v0, v21 & $\{$ Accumulate Intermediate Result $\}$ \\
\hline 41: eor. $16 \mathrm{~b}$ v1, v1, v22 & $\{$ Accumulate Intermediate Result $\}$ \\
\hline
\end{tabular}


friendly binary field multiplication. The recent work by [3] only explores the short 128-bit binary field multiplication.

Table 4. Comparison results of binary field multiplication

\begin{tabular}{cccc}
\hline Algorithm & Architecture & Processor & $\mathbb{F}_{2^{251}}$ \\
\hline KNV [2] & Cortex-A8 & ARMv7 & 385 \\
KNV [2] & Cortex-A9 & ARMv7 & 491 \\
KNV [2] & Cortex-A15 & ARMv7 & 317 \\
Proposed Method (KNP) & Apple-A7 & ARMv8 & 57 \\
\hline
\end{tabular}

\section{Conclusion}

In this paper, we show efficient implementation techniques for binary field multiplication on ARMv8. Our proposed method improves the performance by a factor of 5.5 times than previous techniques on ARMv7. Our future works are efficient binary field elliptic curve cryptography with proposed techniques.

\section{References}

1. D. J. Bernstein. Batch binary edwards. In Advances in Cryptology-CRYPTO 2009, pages 317-336. Springer, 2009.

2. D. Câmara, C. P. Gouvêa, J. López, and R. Dahab. Fast software polynomial multiplication on arm processors using the neon engine. In Security Engineering and Intelligence Informatics, pages 137-154. Springer, 2013.

3. C. P. Gouvêa and J. López. Implementing gcm on armv8. In Topics in CryptologyCT-RSA 2015, pages 167-180. Springer, 2015

4. J. Großschädl, R. M. Avanzi, E. Savaş, and S. Tillich. Energy-efficient software implementation of long integer modular arithmetic. In Cryptographic Hardware and Embedded Systems-CHES 2005, pages 75-90. Springer, 2005.

5. A. Karatsuba and Y. Ofman. Multiplication of multidigit numbers on automata. In Soviet physics doklady, volume 7, page 595, 1963.

6. J. López and R. Dahab. High-speed software multiplication in $\mathrm{f} 2 \mathrm{~m}$. In Progress in CryptologyINDOCRYPT 2000, pages 203-212. Springer, 2000.

7. L. B. Oliveira, D. F. Aranha, C. P. Gouvêa, M. Scott, D. F. Câmara, J. López, and R. Dahab. Tinypbc: Pairings for authenticated identity-based non-interactive key distribution in sensor networks. Computer Communications, 34(3):485-493, 2011.

8. H. Seo, Y. Lee, H. Kim, T. Park, and H. Kim. Binary and prime field multiplication for public key cryptography on embedded microprocessors. Security and Communication Networks, 7(4):774-787, 2014.

9. H. Seo, Z. Liu, J. Choi, and H. Kim. Karatsuba-block-comb technique for elliptic curve cryptography over binary fields. Security and Communication Networks, 2015 . 
10. M. Shirase, Y. Miyazaki, T. Takagi, and D.-G. HAN. Efficient implementation of pairing-based cryptography on a sensor node. IEICE transactions on information and systems, 92(5):909-917, 2009.

11. Steve Ranger. Internet of things and wearables drive growth for ARM. Available for download at http://www.zdnet.com/article/ internet-of-things-and-wearables-drive-growth-for-arm/, Apr. 2014. 